\title{
The Influence of Natural Crack Distribution Uniformity on the Breakdown Pressure and Fracture Mode
}

\author{
PENG Peihuo 1,2,a \\ ${ }^{1}$ School of Science, Beijing University of Civil Engineering \& Architecture, Beijing 102612, China \\ ${ }^{2} S c h o o l$ of Mechanics and Civil Engineering, China University of Mining \& Technology, Beijing \\ 100083, China \\ apph773@bucea.edu.cn
}

\begin{abstract}
Keywords: Distribution uniformity index (DUI); Bonded Particle Method (BPM); PFC3D numerical model; Breakdown pressure; Fracture mode

Abstract The spatial distribution of the hydraulic fractures is mainly controlled by two processes of crack initiation and propagation. It is the premise of predicting the geometric structure of fracture network to study the mechanism of crack initiation of rock. There are a large number of initial micro cracks in the shale reservoir, the presence of these micro cracks weakens the integrity of shale, it must have an important influence on the initiation and propagation of hydraulic fractures. The numerical model of simulating the breakdown pressure and fracture mode of low permeability fractured reservoir is established based on the calculation method of distribution uniformity index. The influence of initial micro crack distribution uniformity on the breakdown pressure and fracture mode is studied. Numerical results show that: the greater the distribution uniformity index of the initial natural micro cracks, the greater the breakdown pressure is; But the accumulative number of tensile and shear fractures and their proportion to the total number of fractures are not affected by the distribution uniformity index of the natural micro cracks.
\end{abstract}

\section{Introduction}

The core technologies for successful exploitation of shale gas are horizontal drilling technology and hydraulic fracturing technology. There is a direct relationship between the morphological structure of fracture network distribution and the recovery efficiency of oil and gas after hydraulic fracturing. The spatial distribution of hydraulic fractures is mainly controlled by two processes of crack initiation and expansion. Therefore, studying the initiation mechanism of hydraulic fracture in shale reservoir is the premise of understanding the morphological structure of fracture network. There is a certain blindness that the fracturing effect is increased only by increasing the injection pressure, or increasing the displacement of the fracturing fluid, or other empirical methods when hydraulic fracturing is performed on site if the mechanism of hydraulic fracture initiation is not clear. Therefore, the accurate determination of fracture mode and accurate prediction of breakdown pressure are of important guiding significance to the design and successful implementation of fracturing scheme.

Many scholars have done a lot of research on fracture mode and breakdown pressure of hydraulic fracture. In 1957, Hubbert et al proposed a formula for calculating the initiation pressure of rock under the three directional stress around the borehole [1]. In 1967, Haimson et al considered the seepage effect of fracturing fluid in rock, and improved the calculation formula of breakdown pressure [2]. And other crack initiation pressure calculation methods [3,4] are based on the isotropic continuum model, these models do not take into account the effects of natural micro crack distribution. In recent years, many scholars have realized that the natural micro fractures of complex distribution are widespread in shale and other low permeable reservoirs. Shale reservoirs experienced different geological movements during the long process of formation, and formed different kinds of natural fractures. The electron microscopic characteristics demonstrate that there are a large number of micro-cracks in shale. These micro cracks or holes are honeycombed or randomly distributed, and the pore structure and crack distribution are also different in various shale reservoir [5-7]. The distribution uniformity of initial micro fractures leads to a strong anisotropy in shale, the crack initiation mechanism and propagation model of hydraulic fracture are more 
complicated. Therefore, the calculation model of crack initiation based on the traditional elastic isotropic medium cannot meet the needs of hydraulic fracturing engineering practice of shale reservoir.

A method for calculating the distribution uniformity index (DUI) of natural micro cracks is presented in this paper. And using PFC3D numerical calculation platform and built-in FISH language, a numerical model for breakdown pressure and fracture model of fractured reservoirs with low permeability is established. The influence of initial micro crack distribution uniformity index on breakdown pressure and fracture model was studied quantitatively. Numerical results show that: when the numerical model of hydraulic fracturing considers the existence of natural micro cracks, the breakdown pressure and fracture model are different from those without initial natural micro cracks.

\section{Numerical Model}

To simulate and analyze hydraulic fracturing characteristics of shale samples in micro scale range, a three-dimensional numerical model is established by using PFC3D software based on Bonded Particle Method (BPM). In the process of generating numerical sample, an assembly consisting of larger particles is first built, and then, the contact force of each particle is tested. These particles are deleted if the maximum contact force of a particle is less than one-tenth the average contact force of the entire sample. Smaller particles are then inserted in this porous sample, and the particles whose contact forces are sufficiently small are deleted according to the same rule. This process continues until a sample with sufficiently low porosity is created.

2.1 Calculation Parameters Each side length $a$ of the PFC3D model is $100 \mathrm{~mm}$, where the diameter $b$ of a borehole is $10 \mathrm{~mm}$, i.e., ten times smaller than $a$, to reduce the stress concentration effect of in situ stress application, and the borehole axis is consistently perpendicular to the outer plane of the model. The numerical models are shown in Fig. 1 and Fig. 2. To provide parameters for the numerical modelling of hydraulic fracturing, a few shale specimens that were sampled from the Longmaxi Formation in the Sichuan Basin in the southwest of China [8] were tested. The values of mechanical properties are listed in Table 1. In order to avoid the influence of horizontal stress difference, the maximum horizontal stress, the minimum horizontal stress and the vertical stress are equal in the numerical calculation, all of which are $10 \mathrm{MPa}$.

2.2 Calculation Method of Initial Crack DUI In PFC3D numerical simulation of fracturing process, the breaking of parallel bonds represents the initiation and propagation of hydraulic fractures. In this paper, the crack initiation mechanism of hydraulic fracture is studied by statistical analysis of the accumulative number and spatial distribution of broken parallel bonds. A method for calculating the uniformity index of fracture distribution is proposed below. The influence of the initial natural micro crack distribution uniformity on the breakdown pressure and fracture mode of hydraulic fracturing is analyzed by calculating the cumulative number of parallel bond breaking events and the induced fracture distribution uniformity index.

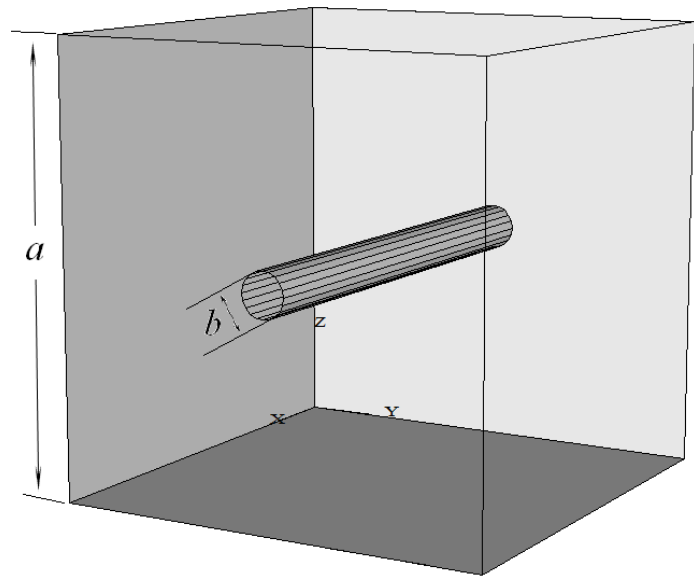

Fig. 1 Numerical calculation model (3D view)

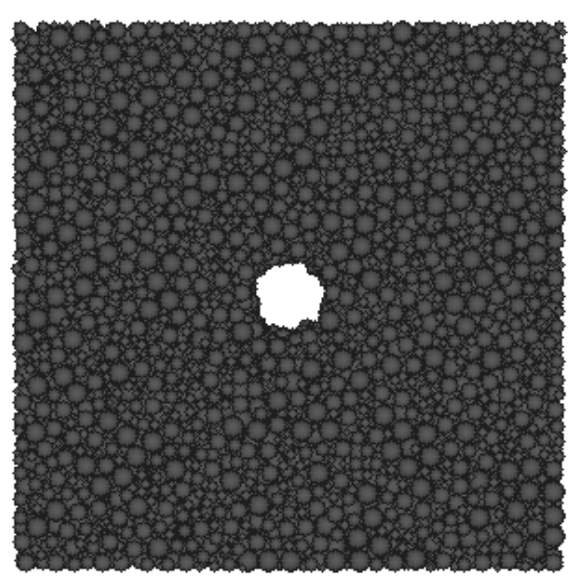

Fig. 2 PFC3D numerical model (Side view) 
Table 1 Mechanical properties of the shale of the Longmaxi Formation in the Sichuan Basin

\begin{tabular}{ccccc}
$\begin{array}{c}\text { Density } \\
{\left[\mathrm{g} \cdot \mathrm{cm}^{-3}\right]}\end{array}$ & $\begin{array}{c}\text { Uniaxial compressive } \\
\text { strength }[\mathrm{MPa}]\end{array}$ & $\begin{array}{c}\text { Tensile strength } \\
{[\mathrm{MPa}]}\end{array}$ & $\begin{array}{c}\text { Elastic modulus } \\
{[\mathrm{GPa}]}\end{array}$ & $\begin{array}{c}\text { Poisson's } \\
\text { ratio }\end{array}$ \\
\hline 2.6 & 70 & 3.2 & 25 & 0.27 \\
\hline
\end{tabular}

In the fracturing simulation, the spatial position information of every broken parallel bond is recorded. When the simulation is finished, the distribution uniformity index (DUI) is calculated according to the following method. The model sample is divided into $8^{n} \quad(n=0,1,2, \ldots)$ small cubes (as shown in Fig. 3). The cube for each side is $100 / 2^{n} \mathrm{~mm}$. The position of each broken parallel bond must be located in one of the $8^{n}$ cubes. In these cubes, the number of cubes that include a broken parallel bond is recorded as $g(n)$.

$$
f(n)=\frac{g(n)}{8^{n}}, n=0,1,2, \ldots
$$

The function $f(n)$ is plotted in the coordinate system in Fig. 4; the ratio of the area surrounded by the curve $f(n)$ and curve $f_{0}(n)$ divided by the area surrounded by the curve $f_{1}(n)$ and curve $f_{0}(n)$ is defined as the Distribution Uniformity Index (DUI), where the functions $f_{0}(n)$ and $f_{1}(n)$ are as follows:

$$
f_{0}(n)=\frac{1}{8^{n}} \quad, \quad f_{1}(n)=1 .
$$

From the definition of the DUI, when the distribution of the cracks is highly concentrated, even if the cube is divided into small segments, all of the cracks are always located in a cube in this extreme case:

$$
f(n)=f_{0}(n)=\frac{1}{8^{n}} .
$$

In this case, DUI $=0$.

Another extreme case is when the crack distribution is absolutely uniform; even if the cube is divided into small segments, all of the cubes always contain cracks, namely:

$$
f(n)=f_{1}(n)=1 .
$$

In this case, DUI $=1$. According to the definition of distribution uniformity index, the range of DUI is between $0 \sim 1$. The smaller the value of DUI, the closer it is to 0 , indicates that the fracture distribution is more concentrated; And the bigger the value of DUI, the closer it is to 1, indicates that the more uniform the fracture distribution is.

Theoretically, the cubic sample can be divided infinitely. However, in the calculation of this paper, the maximum of $n$ is only 6 . If the number $n$ is too large, the volume of the small cube may be smaller than the size of the micro-particles, and then, it is of no practical significance to continue to divide the cube. In the calculation of the DUI, only the areas in front of the vertical line $n=6$ are considered. 


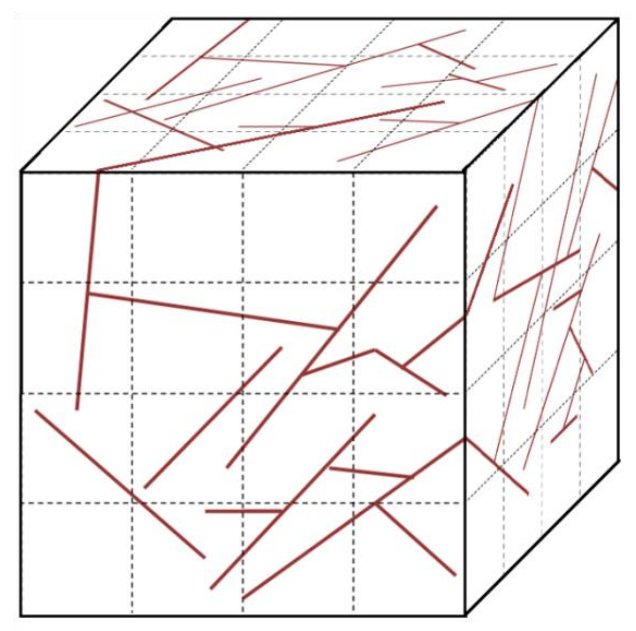

Fig. 3 The specimen was divided $n$ times along the length, width and length direction

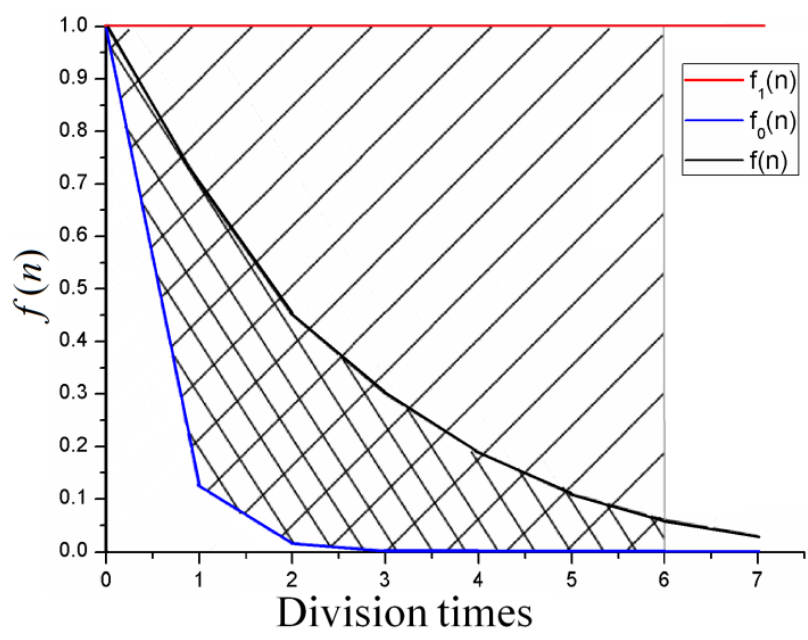

Fig. 4 DUI is the ratio of area surrounded by the curve $f(n)$ and the curve $f_{0}(n)$ and area surrounded by the curve $f_{1}(n)$ and the curve $f_{0}(n)$.

\section{Model Verification}

In order to verify the numerical model, the breakdown pressure measured by the numerical model is compared with the theoretical results. In the hydraulic fracturing simulation, the flow rate of fracturing fluid is set as a constant, and record the change of pressure in borehole at any time. The pressure increases with time at the beginning, then a sharp decline begins when a peak is reached, subsequently stabilize at a certain pressure level, the peak value of the whole pressure curve is the breakdown pressure. The corresponding pressure curve is shown in Fig. 5.

For the sake of simplicity, we assume that the minimum horizontal stress is equal to the maximum horizontal stress in the verification model, the breakdown pressure at six different horizontal stresses is simulated. The numerical results are shown in Table 2.

Table 2 Numerical results of the breakdown pressure

\begin{tabular}{ccccccc}
\hline Horizontal stresses[MPa] & 10 & 11 & 12 & 13 & 14 & 15 \\
\hline Breakdown pressure[MPa] & 11.75 & 12.93 & 12.95 & 14.57 & 15.23 & 16.01 \\
\hline
\end{tabular}

If the rock is assumed to be fully permeable and the pressurization rate is sufficiently low to ensure steady-state conditions during pumping, the relationship between the breakdown pressure $P_{b}$ and horizontal stress is as follows [9]:

$$
P_{b}=\frac{3 S_{h}-S_{H}-\alpha(1-2 \mu) /(1-\mu) p_{f}+T}{2-\alpha(1-2 \mu) /(1-\mu)}, 0 \leq \alpha \leq 1
$$

where $T$ is the rock tensile strength, $p_{f}$ is the pressure in the fracture or in the pores where the fracture is formed, $S_{H}$ is the major horizontal stress and $S_{h}$ is the minor horizontal stress, $\alpha$ is the poroelastic coefficient and $\mu$ is Poisson's ratio.

Based on the theoretical formula, the breakdown pressure at 6 different horizontal stresses is calculated when the poroelasticity coefficient is 0.29 . The value of the pressure $p_{f}$ for the fully 
permeable case in the theoretical calculation is taken from the pressure of the fluid cell at the crack initiation location in the numerical model.

A comparison between the analytical results and the numerical results is shown in Fig. 6. It can be seen from Fig. 6 that the numerical results is in good agreement with the analytical results when the poroelasticity coefficient is 0.29 . The breakdown pressure obtained by the BPM is consistent with the theoretical results. This indicates that the BPM is feasible for modelling the fracturing process.

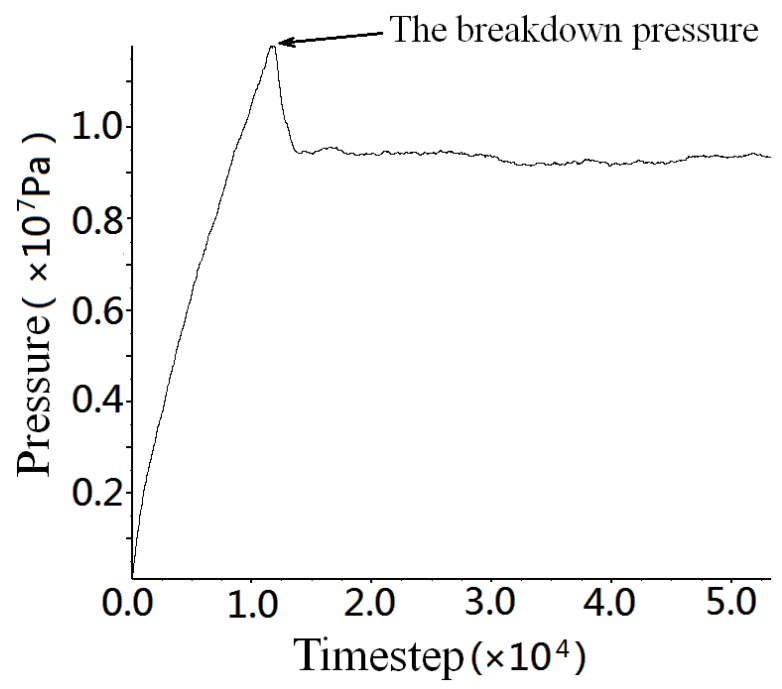

Fig. 5 Pressure varying with timestep during the fracturing simulation

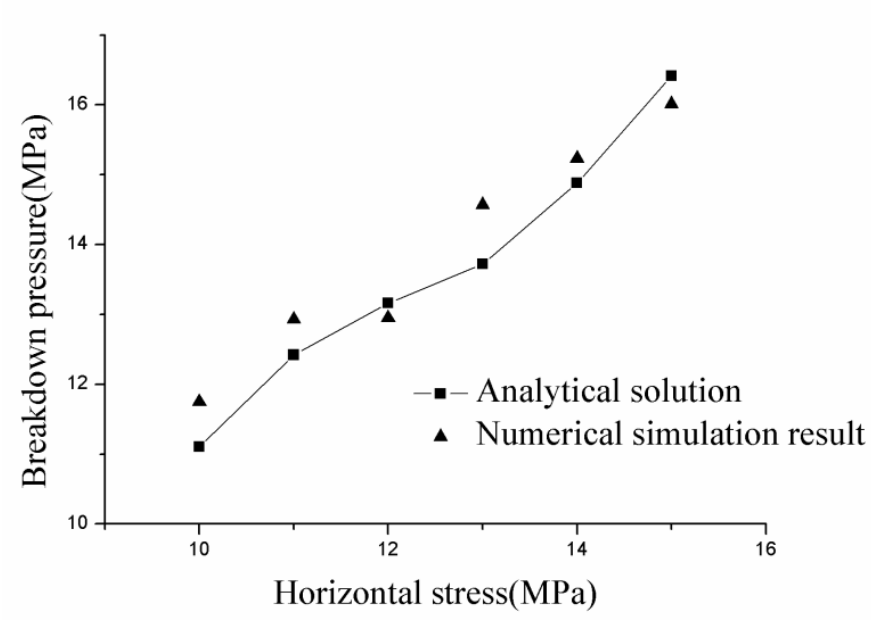

Fig. 6 Breakdown pressure varying with horizontal stress

\section{Case Study and Result Analysis}

In the PFC3D numerical model, the bond between particles breaks down, indicating the formation of micro cracks. The process of bonds breaking and converging in succession indicates the initiation and propagation of macroscopic cracks. In order to study the influence of initial crack distribution on breakdown pressure and fracture model, on the basis of the verification model in the previous section, the initial natural crack distribution in the numerical model is simulated by deleting some randomly distributed parallel bonds in the sample. The program of randomly deleting parallel bonds repeats several times. For each generated numerical model with random initial cracks, the fracture distribution uniformity index is calculated, 6 models with equal number of micro cracks but unequal distribution uniformity are found out, then carry on the fracturing simulation. The breakdown pressure of each model and the accumulative number of tensile fractures and shear fractures are recorded. The numerical results are shown in Fig. 7 and Fig. 8.

4.1 Breakdown Pressure Fig. 7 shows the relationship between the breakdown pressure and the natural fracture distribution uniformity index for the 6 models with the same initial crack number but the uniformity of fracture distribution is not equal. As can be seen from Fig. 7, with the initial crack distribution uniformity index increasing, that is, the micro crack distribution is more uniform, the breakdown pressure has a gradually increasing trend; The smaller the distribution uniformity index of the initial natural crack is, namely the micro crack distribution is more concentrated, and the smaller the breakdown pressure is. Thus, when the minimum horizontal stress is the same as the maximum horizontal stress, the breakdown pressure is obviously related to the uniformity of the natural micro crack distribution in the rock medium.

4.2 Fracture Mode Fig. 8 shows the total number of hydraulic fractures, the accumulative number of tensile fractures and shear fractures formed in the numerical model when the pressure 
reaches the instant of breakdown pressure during the fracturing simulation. As can be seen from Fig. 8 , the total number of cracks does not exhibit a monotonic increase or decrease monotonically with the different initial distribution uniformity of the micro cracks. Moreover, the number of tensile fractures and shear fractures and their proportion to the total number of fractures have no obvious trend with the change of distribution uniformity index. Therefore, there is no obvious correlation between the fracture mode and the initial crack distribution uniformity in the rock medium.

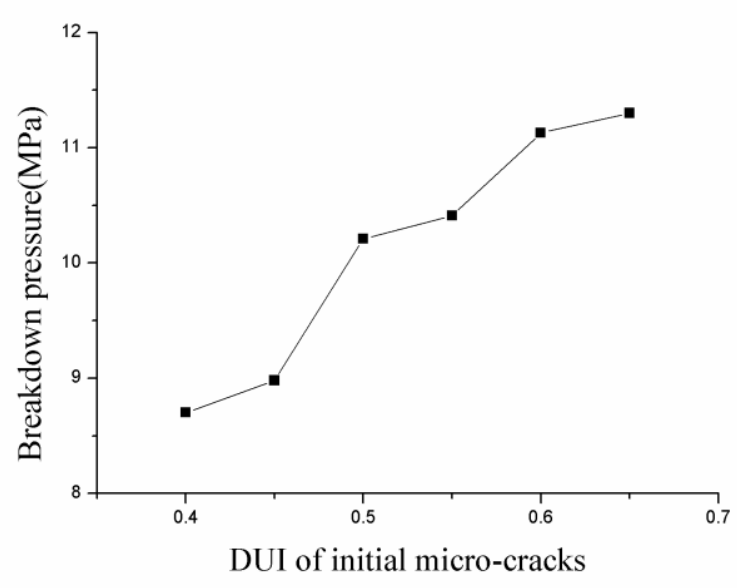

Fig. 7 The relationship between breakdown pressure and initial crack distribution uniformity index

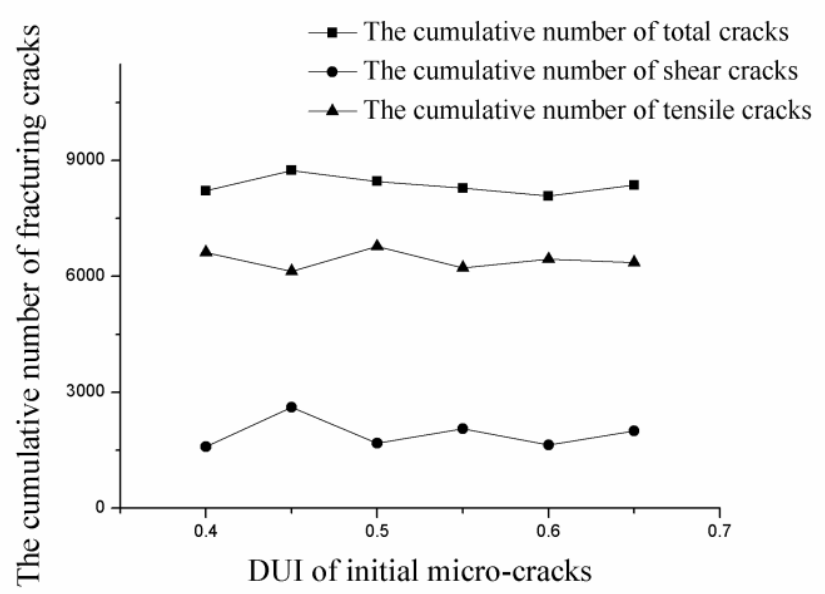

Fig. 8 The accumulative number of hydraulic fractures and the number of tensile break and shear break

\section{Conclusion}

The electron microscopic characteristics demonstrate that there are a large number of micro-cracks in shale. The spatial distribution and structure of these microcracks are also distinctly different in various shales. The influence of the spatial distribution of initial micro cracks on the breakdown pressure and fracture mode of hydraulic fracturing is studied with the PFC3D numerical model based on Bonded Particle Method. The results are summarized as follows:

(1) If the amount of initial micro cracks is same, when the minimum horizontal stress is equal to the maximum horizontal stress, the larger the initial crack distribution uniformity index is, the greater the breakdown pressure is; On the contrary, the breakdown pressure is smaller.

(2) From the results of numerical simulation, the accumulative number of tensile fractures and shear fractures and their proportion to the total number of fractures are not obviously related to the distribution uniformity of initial micro cracks in shale.

\section{Acknowledgements}

This work was financially supported by the Science Project Foundation of Beijing Municipal Education Commission (SQKM201710016013), Science Research Foundation of BUCEA (00331616030).

\section{References}

[1] Hubbert M K, Willis D G. Mechanics of hydraulic fracturing. Trans AIME. 1957;210:153-63.

[2] Haimson B, Fairhurst C. Initiation and extension of hydraulic fractures in rocks. Society of Petroleum Engineers Journal. 1967;7(3):310-8. 
[3] Hossain M M, Rahman M K, Rahman S S. Hydraulic fracture initiation and propagation: roles of wellbore trajectory, perforation and stress regimes. Journal of Petroleum Science and Engineering. 2000;27(3):129-49.

[4] Ito Takatoshi. Effect of pore pressure gradient on fracture initiation in fluid saturated porous media rock. Engineering Fracture Mechanics. 2008;75:1753-62.

[5] Zeng W, Zhang J, Ding W, et al. Fracture development in Paleozoic shale of Chongqing area (South China). Part one: Fracture characteristics and comparative analysis of main controlling factors. Journal of Asian Earth Sciences. 2013;75:251-66.

[6] Dong T, Harris N B, Ayranci K, et al. Porosity characteristics of the Devonian Horn River shale, Canada: Insights from lithofacies classification and shale composition. International Journal of Coal Geology. 2015;141-142:74-90.

[7] Liang L, Xiong J, Liu X. Mineralogical, microstructural and physiochemical characteristics of organic-rich shales in the Sichuan Basin, China. Journal of Natural Gas Science and Engineering. 2015;26:1200-12.

[8] Yang J, Fu Y, Chen H, et al. Rock mechanical characteristics of shale reservoirs. Natural Gas Industry. 2012; 32(7):12-4+100.

[9] Fjar E, Holt R M, Horsrud P, et al. Petroleum related rock mechanics: 2nd edition. Amsterdam, The Netherlands: Elsevier Science; 2008. 